Angew Chem Int Ed Engl. 2018 May 22; 57(21): 6212-6215. doi:10.1002/anie.201802269.

\title{
Modulating the Folding Landscape of Superoxide Dismutase 1 with Targeted Molecular Binders
}

\author{
David N. Bunck, Beatriz Atsavapranee, Anna K. Museth, David VanderVelde, and James R. \\ Heath \\ Division of Chemistry and Chemical Engineering California Institute of Technology1200 East \\ California Boulevard, MC 172-27 (USA)
}

\begin{abstract}
Amyotrophic lateral sclerosis, or Lou Gehrig's disease, is characterized by motor neuron death, with average survival times of two to five years. One cause of this disease is the misfolding of superoxide dismutase 1 (SOD1), a phenomenon influenced by point mutations spanning the protein. Herein, we used an epitope-specific high-throughput screen to identify a peptide ligand that stabilizes the SOD1 native conformation and accelerates its folding by a factor of 2.5. This strategy may be useful for fundamental studies of protein energy landscapes as well as designing new classes of therapeutics.
\end{abstract}

\section{Keywords}

high-throughput screening; neurodegenerative diseases; peptides; protein folding; superoxide dismutase 1

\begin{abstract}
Superoxide dismutase 1 (SOD1) is a cytosolic protein that mitigates oxidative stress by converting $\mathrm{O}_{2}{ }^{-}$-into $\mathrm{H}_{2} \mathrm{O}_{2}$ and $\mathrm{O}_{2}$. While the wild type (WT) structure is highly stable with denaturation at approximately $95^{\circ} \mathrm{C}$, destabilizing point mutations increase its propensity to misfold and aggregate, ultimately leading to amyotrophic lateral sclerosis (ALS). ${ }^{[1-5]}$ ALS is characterized by motor neuron death, ${ }^{[6]}$ with a survival time of two to five years after diagnosis and only two FDA-approved treatments with marginal benefit. ${ }^{[7]}$ Over 160 point mutations spanning 90 residues on SOD1 are linked to ALS, ${ }^{[8]}$ complicating the development of general therapeutics that address its misfolding. ${ }^{[9]}$ In vitro studies indicate that both the unfolded and native states lie on the aggregation pathway, ${ }^{[2,4]}$ suggesting that stabilizing the ground-state structure while accelerating folding might address underlying disease mechanisms. ${ }^{[10,11]}$ Epitope-specific antibodies and their antigen-recognition regions as well as overexpressed heat shock proteins ${ }^{[12]}$ have shown some therapeutic benefit in ALS mouse models. ${ }^{[3,11,13]}$ Small molecules identified in cell-based screens have also mitigated toxicity from SOD1 aggregation in preclinical studies, yet their biological targets remain unknown. ${ }^{[14]}$ We sought to combine the advantages of these approaches by exploring
\end{abstract}

Supporting information and the ORCID identification number(s) for the author(s) of this article can be found under: https://doi.org/ 10.1002/anie.201802269.

Conflict of interest

James Heath is a founder and board member of Indi Molecular, which is seeking to commercialize the PCC technology. 
the small-molecule-targeted stabilization of key SOD1 epitopes so as to modify its folding energy landscape by stabilizing its folded structure while accelerating its folding.

To perform in these roles, the ligands must bind the folded ground-state structure and stabilize the rate-limiting transition state. We focused on the electrostatic loop (amino acids 121-144; Figure 1a, red), which is a target relevant to both functions. Studies of ALScausing mutants by NMR spectroscopy, ${ }^{[15,16]} \mathrm{H} / \mathrm{D}$ exchange, ${ }^{[15,17]}$ and high-resolution Xray diffraction ${ }^{[18-21]}$ indicate consistent destabilization of this loop when even distant mutations are present. ${ }^{[3]}$ Investigations into the relative impact of point mutations on the folding rate and protein stability ${ }^{[22]}$ highlight the importance of this region for the ratelimiting transition state. ${ }^{[23,24]}$ Ligands binding to the loop might accelerate folding while mitigating the effects of mutations on the ground-state structure (Figure 1b).

To identify such ligands, we employed an in situ click screen that furnishes epitope-specific peptides. ${ }^{[25]}$ A peptide containing amino acids $121-141$ representing the electrostatic loop was synthesized with a Leu ${ }^{126}$ | propargyl glycine substitution and a C-terminal biotin assay handle (Figure 1a), and subsequently incubated with a one-bead-one-compound library of cyclic peptides bearing $\mathrm{N}$-terminal azides. For this in situ click screen, library members that bind the target epitope induce a dipolar cycloaddition between the azide and alkyne (without $\mathrm{Cu}^{\mathrm{I}}$ ), decorating hit beads with biotin. Through tandem molecular recognition and enzymatic amplification, these hits were identified and sequenced by Edman degradation.

The screen yielded hits with observable similarity (see the Supporting Information, Figure S2), which were tested for binding against the full-length protein (Figure S4). The ligand with the best binding affinity, containing the variable region sequence $\mathrm{HGG}^{4 \mathrm{~F}} \mathrm{FQ}\left({ }^{4 \mathrm{~F}} \mathrm{~F}=4\right.$ fluorophenylalanine) (Figure 2), bound the metalated (holo) protein with $\mathrm{EC}_{50}=8.0 \mu \mathrm{m}$ (Figure S6) and the non-metalated (apo) protein with $\mathrm{EC}_{50}=0.94 \mu \mathrm{m}$ (Figure S7). This binding difference may be due to the increased disorder of the electrostatic loop in the apo form, which more closely resembles the conformations adopted by the synthetic epitope. The peptide also bound three ALS-causing mutants containing destabilized electro-static loops, G85R, D90A, and G93A, with similar affinities (Figure S8). ${ }^{1} \mathrm{H}-{ }^{15} \mathrm{~N}$ HSQC NMR spectroscopy confirmed selective binding of the peptide to the electrostatic loop on both the holo (Figure 3 and Figures S12-S15) and apo (Figures S16-S20) variants. Statistical analysis of these spectra revealed that equimolar concentrations of ligand with holo SOD1 caused resonances associated with the loop to either change intensity, shift, or disappear altogether. At increased ligand concentrations, additional residues near the loop and at the dimer interface were shifted. Connolly maps of SOD1 revealed that those residues at the dimer interface are not solvent-exposed, and so perturbations in this region are likely allosteric effects. ${ }^{[26]}$ For apo SOD1, perturbations at 1 equiv were similar to those observed at 2 equiv with holo SOD1, which is consistent with the expected occupancy of $\mathrm{HGG}^{4 \mathrm{~F}} \mathrm{FQ}$ on each protein variant (Figure S20).

We employed dynamic light scattering (DLS) analysis to gain insight into the effects of binding to the electrostatic loop. The hydrodynamic radius $\left(l_{\mathrm{h}}\right)$ of apo WT SOD1 decreased with the addition of $\mathrm{HGG}^{4 \mathrm{~F}} \mathrm{FQ}$ from $3.68 \pm 0.26 \mathrm{~nm}$ to $2.88 \pm 0.07 \mathrm{~nm}$ (Figure 4). The resulting complex was smaller than the holo WT protein $(3.32 \pm 13 \mathrm{~nm})$, suggesting that 
ligand binding induces a tightening of the peripheral structure of the protein, which is disordered without metal (Figure S21a). Higher-amplitude structural fluctuations were also observed in the absence of ligand (Figure S21b). Thus ligation both reduced the $I_{\mathrm{h}}$ of apo SOD1 and narrowed the width of those fluctuations (Figure 4). Similar size decreases were not observed with holo WT SOD1 (Figures S21, S22, and S29), implying that the more stabilized variant is less affected by ligand binding. These observations are consistent with ${ }^{1} \mathrm{H}-{ }^{15} \mathrm{~N}$ HSQC spectra, where ligand titration perturbed a narrow band of residues that intersects the $\mathrm{z}$ axis of the $\beta$-barrel. With increasing $\mathrm{HGG}^{4 \mathrm{~F}} \mathrm{FQ}$ concentration, the peak intensities across the spectrum increased, indicating reduced conformational freedom along the amide backbone of the protein. Ligand-induced decreases in $I_{\mathrm{h}}$ were also observed with G93A and D90A (Figure S23) SOD1. Apo G93A was larger than WT $(4.27 \pm 0.40 \mathrm{~nm})$, which highlights its more destabilized structure. ${ }^{[27]}$ Notably, ligand binding to this mutant reduced its $l_{\mathrm{h}}$ to match the WT structure $(3.04 \pm 0.19 \mathrm{~nm})$. An additional impurity at higher $\iota_{\mathrm{h}}$ deriving from $\mathrm{HGG}^{4 \mathrm{~F}} \mathrm{FQ}$ was also observed in DLS traces (Figure S24). Because of the sharply increased light scattering intensity with an increasing hydrodynamic radius (I a $I_{\mathrm{h}}{ }^{6}$ ), this peak represents less than $0.01 \%$ of these samples/. No additional species were observed in these mixtures by SEC-UV and ${ }^{1} \mathrm{H} /{ }^{19} \mathrm{~F}$ NMR analysis (see the Supporting Information and Figures S10, S11, and S24-S29); we therefore conclude that this low-abundance impurity is not involved with the phenomena described here. These measurements indicate that binding the electrostatic loop stabilizes the hydrodynamic structure of apo WT SOD1 and its mutants through allosteric interactions connecting the electrostatic loop and the $\beta$ barrel.

To understand the impact of ligand binding on the rate-limiting transition state of folding, we generated a chevron plot for SOD1 (Figure 5). ${ }^{[28]}$ Here, the folding $\left(k_{\mathrm{f}}\right)$ and unfolding $\left(k_{\mathrm{u}}\right)$ rates were plotted against [ $\left.\mathrm{GdmCl}\right]$, generating a characteristic $\mathrm{V}$-shaped plot where extrapolation to the intercept furnishes the rates at zero denaturant concentration $\left(k_{\mathrm{f}, 0}\right.$ and $k_{\mathrm{u}, 0}$ ). A mutant whose surface cysteines were removed (pWT, C6A/C111S) was employed in its apo form for these studies. SOD1 contains one Trp on each monomer (and no Tyr), enabling this measurement by monitoring changes in fluorescence (Figure S31) ${ }^{[29]}$ The protein was first denatured with $\mathrm{GdmCl}$ and then diluted in buffer containing the ligand (Figure S29). We found consistent folding rate increases across a range of $\mathrm{GdmCl}$ concentrations. Notably, the slopes of $\ln \left(k_{\mathrm{f}}\right)$ versus $[\mathrm{GdmCl}]$ were similar with $\left(m_{\mathrm{f}, \mathrm{L}}=\right.$ -2.74) and without $\left(m_{\mathrm{f}}=@ 2.52\right)$ ligand, indicating that the basic rate-limiting transition state remains unchanged. Extrapolation to $[\mathrm{GdmCl}]=0 \mathrm{~m}$ revealed a 2.5 -fold increase in the folding rate with ligand $(p<0.005)$. At $[\mathrm{GdmCl}]>1.5 \mathrm{~m}$, we measured the unfolding rate with Trp fluorescence by adding denaturant directly to the folded protein (Figure S29). Unfolding in the absence of ligand also revealed a linear relationship between $\ln \left(k_{\mathrm{u}}\right)$ and [GdmCl], where $k_{\mathrm{u}, 0}$ was reduced by a factor of 1.2 in the presence of ligand; however, this difference was not statistically significant $(p<0.20)$. Knowledge of $k_{\mathrm{f}, 0}$ and $k_{\mathrm{u}, 0}$ yields the $K_{\text {eq }}$ and $\Delta \mathrm{G}$ values of folding (Table S2). In the absence of ligand, $\Delta G_{\mathrm{f}}=-2.84 \mathrm{kcal} \mathrm{mol}^{-1}$, which drops to $\Delta G_{\mathrm{f}}=-3.49 \mathrm{kcal} \mathrm{mol}^{-1}$ upon $\mathrm{HGG}^{4 \mathrm{~F}} \mathrm{FQ}$ binding. Comparing the change in $\Delta G$ for the folded $\left(\Delta \Delta G_{\mathrm{f}}\right)$ and transition-state $\left(\Delta \Delta G^{\star}\right)$ structures reveals that nearly $80 \%$ of the stability imparted by $\mathrm{HGG}^{4 \mathrm{~F}} \mathrm{FQ}$ derives from an increasing $k_{\mathrm{f}}$ value as opposed to a reducing $k_{\mathrm{u}}$ value, the latter of which is more common for pharmacological chaperones. ${ }^{[30]}$ 
Moreover, this acceleration represents only a fraction of the overall binding energy available from the ligand (for $K_{\mathrm{D}}=8.01 \mu \mathrm{m}, \Delta G=-6.95 \mathrm{kcal} \mathrm{mol}^{-1}$ ). In preliminary experiments, this phenomenon was also observed with pG93A SOD1, where $\mathrm{HGG}^{4 \mathrm{~F}} \mathrm{FQ}$ accelerated folding by a factor of 1.5 at $[\mathrm{GdmCl}]=0.5 \mathrm{~m}$ (Figure $\mathrm{S} 23$ ). The relatively smaller influence on this mutant might derive from increased destabilization of the electro-static loop upon mutation. Overall, increasing $k_{\mathrm{f}, 0}$ without perturbing $k_{\mathrm{u}, 0}$ indicates that targeting the electrostatic loop lowers the barrier to folding with concomitant stabilization of the native state.

In conclusion, we have developed a targeted ligand designed to modulate the folding energy landscape of SOD1. The cyclic peptide $\mathrm{HGG}^{4 \mathrm{~F}} \mathrm{FQ}$ stabilizes and tightens the ground-state structure, while also accelerating folding by reducing the activation barrier to the native state. Even with a modest binding affinity, its influence on the folding rate is significant. The majority of chaperones function as holdases, preventing off-pathway aggregation, rather than as foldases, which accelerate folding. Foldases, which are both ATP-dependent and independent, exhibit a range of rate enhancements from 1.5 to $50{ }^{[31]}$ In this work, a peptide that is one to two orders of magnitude smaller than these chaperones improved the folding rate by a factor of 2.5 . We speculate that the outsized impact of $\mathrm{HGG}^{4 \mathrm{~F}} \mathrm{FQ}$ derives from its targeted recognition of the electrostatic loop, a key component of the SOD1 folding trajectory. An open question is the function of these types of stabilizing ligands within more complex environments. Chaperones in vivo operate in high concentrations of adventitious protein, and we intend to leverage the molecularity of these reagents to understand how folding rates might be engineered in these settings.

\section{Supplementary Material}

Refer to Web version on PubMed Central for supplementary material.

\section{Acknowledgements}

This work was supported by the Institute for Collaborative Biotechnologies (W911NF-09-D-0001) from the U.S. Army Research Office and the Jean Perkins Foundation. Instrumentation was provided by the Center for Catalysis and Chemical Synthesis, the Protein Expression Center, and the Center for the Chemistry of Cellular Signaling at Caltech.D.N.B. acknowledges an NIH Postdoctoral Fellowship. B.A. acknowledges Mary Vodopia and Laurence J. Stuppy SURF fellowships.

\section{References}

[1]. Bosco DA et al., Nat. Neurosci 2010, 13, 1396-1403. [PubMed: 20953194]

[2]. Redler RL, Dokholyan NV, Progress in Molecular Biology and Translational Science, Elsevier, Amsterdam, 2012, pp. 215-262.

[3]. Rotunno MS, Bosco DA, Front. Cell. Neurosci 2013, https://doi.org/10.3389/fncel.2013.00253.

[4]. Robberecht W, Philips T, Nat. Rev. Neurosci 2013, 14, 248-264. [PubMed: 23463272]

[5]. Ivanova MI, Sievers SA, Guenther EL, Johnson LM,Winkler DD, Galaleldeen A, Sawaya MR, Hart PJ, Eisenberg DS, Proc. Natl. Acad. Sci. USA 2014, 111, 197-201. [PubMed: 24344300]

[6]. Ayers JI, Fromholt SE, O’Neal VM, Diamond JH, Borchelt DR, Acta Neuropathol 2016, 131, 103-114. [PubMed: 26650262]

[7]. Cheah BC, Vucic S, Krishnan AV, Kiernan MC, Curr. Med. Chem 2010, 17, 1942-1199. [PubMed: 20377511]

[8]. Abel O, Powell JF, Andersen PM, Al-Chalabi A, Hum. Mutat 2012, 33, 1345-1351. [PubMed: 22753137] 
[9]. Cudkowicz ME, McKenna-Yasek D, Sapp PE, Chin W, Geller B, Hayden DL, Schoenfeld DA, Hosler BA, Horvitz HR, Brown RH, Ann. Neurol 1997, 41, 210-221. [PubMed: 9029070]

[10]. Seetharaman SV, Prudencio M, Karch C, Holloway SP, Borchelt DR, Hart PJ, Exp. Biol. Med 2009, 234, 1140-1154.

[11]. Subado J, Casanovas A, Rodrigo H, Arqué G, Esquerda JE, Neuroscience 2015, 310, 38-50. [PubMed: 26384962]

[12]. Nagy M, Fenton WA, Li D, Furtak K, Horwich AL, Proc. Natl. Acad. Sci. USA 2016, 113, 5424 5428. [PubMed: 27114530]

[13]. Gros-Louis F, Soucy G, Larivière R, Julien J-P, J. Neurochem 2010, 113, 1188-1199. [PubMed: 20345765]

[14]. Zhang Y, Benmohamed R, Zhang W, Kim J, Edgerly CK, Zhu Y, Morimoto RI, Ferrante RJ, Kirsch DR, Silverman RB, ACS Med. Chem. Lett 2012, 3, 584-587. [PubMed: 22837812]

[15]. Shipp EL, Cantini F, Bertini I, Valentine JS, Banci L, Biochemistry 2003, 42, 1890-1899. [PubMed: 12590575]

[16]. Banci L, J. Biol. Chem 2005, 280, 35815-35821. [PubMed: 16105836]

[17]. Molnar KS, Karabacak NM, Johnson JL, Wang Q, Tiwari A, Hayward LJ, Coales SJ, Hamuro Y, Agar JN, J. Biol. Chem 2009, 284, 30965-30973. [PubMed: 19635794]

[18]. Antonyuk S, Elam JS, Hough MA, Strange RW, Doucette PA, Rodriguez JA, Hayward LJ, Valentine JS, Hart PJ, Hasnain SS, Protein Sci 2005, 14, 1201-1213. [PubMed: 15840828]

[19]. Cao X et al., J. Biol. Chem 2008, 283, 16169-16177. [PubMed: 18378676]

[20]. Galaleldeen A, Strange RW, Whitson LJ, Antonyuk SV, Narayana N, Taylor AB, Schuermann JP, Holloway SP, Hasnain SS, Hart PJ, Arch. Biochem. Biophys 2009, 492, 40-47. [PubMed: 19800308]

[21]. Seetharaman SV et al., Biochemistry 2010, 49, 5714-5725. [PubMed: 20515040]

[22]. Nordlund A, Oliveberg M, Proc. Natl. Acad. Sci. USA 2006, 103, 10218-10223. [PubMed: 16798882]

[23]. Banci L, Bertini I, Cramaro F, Del Conte R, Viezzoli MS, Biochemistry 2003, 42, 9543-9553. [PubMed: 12911296]

[24]. Nordlund A, Oliveberg M, HFSP J 2008, 2, 354-364. [PubMed: 19436494]

[25]. Das S et al., Angew. Chem. Int. Ed 2015, 54, 13219-13224; Angew. Chem. 2015, 127, 1341713422.

[26]. Khare SD, Dokholyan NV, Proc. Natl. Acad. Sci. USA 2006, 103, 3147-3152. [PubMed: 16488975]

[27]. Pratt AJ et al., Proc. Natl. Acad. Sci. USA 2014, 111, E4568-E4576. [PubMed: 25316790]

[28]. Lindberg MJ, Normark J, Holmgren A, Oliveberg M, Proc. Natl. Acad. Sci. USA 2004, 101, 15893-15898. [PubMed: 15522970]

[29]. Rumfeldt JAO, Lepock JR, Meiering EM, J. Mol. Biol 2009, 385, 278-298. [PubMed: 18951903]

[30]. Convertino M, Das J, Dokholyan NV, ACS Chem. Biol 2016, 11, 1471-1489. [PubMed: 27097127]

[31]. Hartl FU, Bracher A, Hayer-Hartl M, Nature 2011, 475, 324-332. [PubMed: 21776078] 
a.
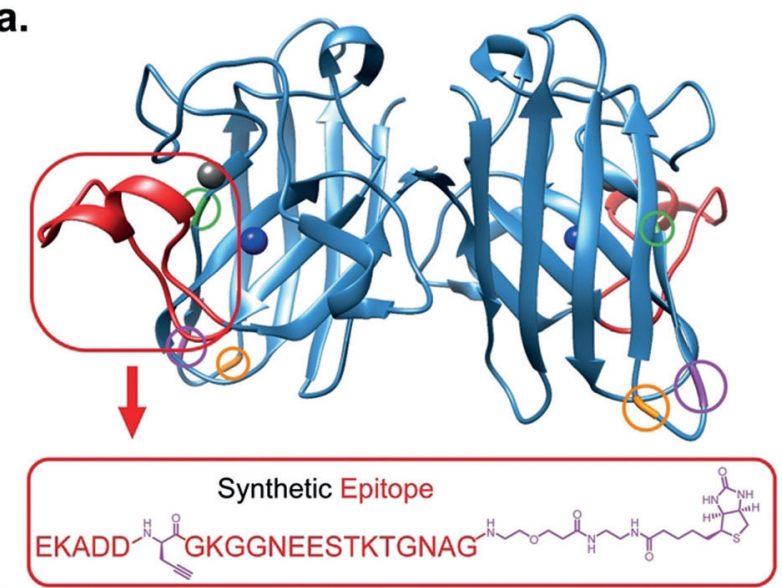

b.

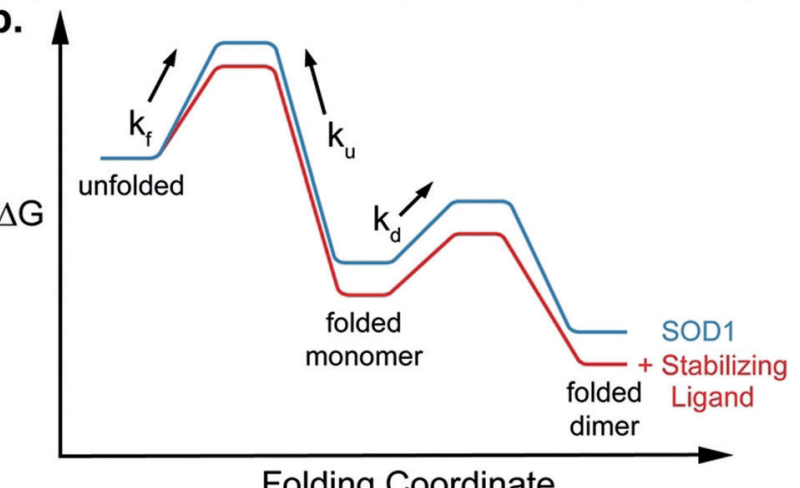

Figure 1.

a) SOD1 is a $C_{2}$-symmetric dimer with a copper active site (blue) and a zinc cofactor (gray). The electrostatic loop (red) is destabilized across several ALS-causing mutations, including G85R (green), D90A (purple), and G93A (orange). b) The apo protein exhibits two-state folding, with fast dimerization relative to folding (PDB No. 2V0A). 


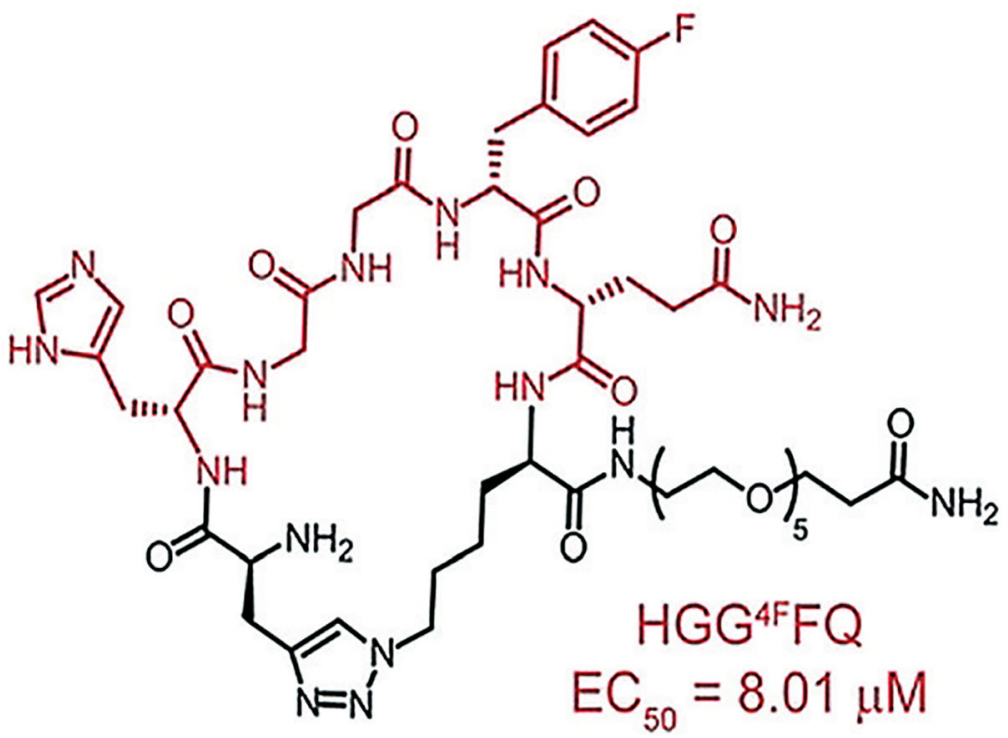

Figure 2.

The $\mathrm{HGG}^{4 \mathrm{~F}} \mathrm{FQ}$ peptide macrocycle ligand from the in situ click screen against the synthesized electrostatic loop epitope of SOD1. 

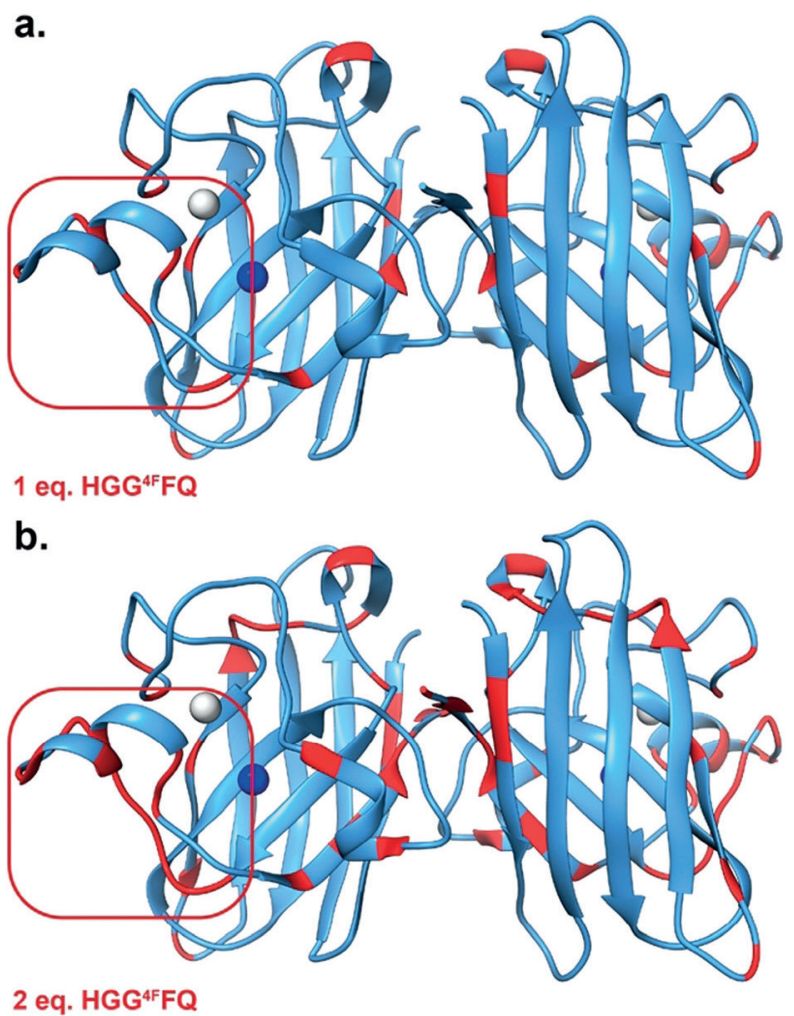

Figure 3.

a) ${ }^{1} \mathrm{H}-{ }^{15} \mathrm{~N}$ HSQC NMR spectroscopy on holo WT SOD1 with $\mathrm{HGG}^{4 \mathrm{~F}} \mathrm{Q}$ reveals localized binding around the electrostatic loop (red square) at 1 equiv ligand. b) Additional residues are perturbed at 2 equiv $\mathrm{HGG}^{4 \mathrm{~F}} \mathrm{FQ}$ through allosteric effects. Ligand equivalents are relative to the monomer subunit. 


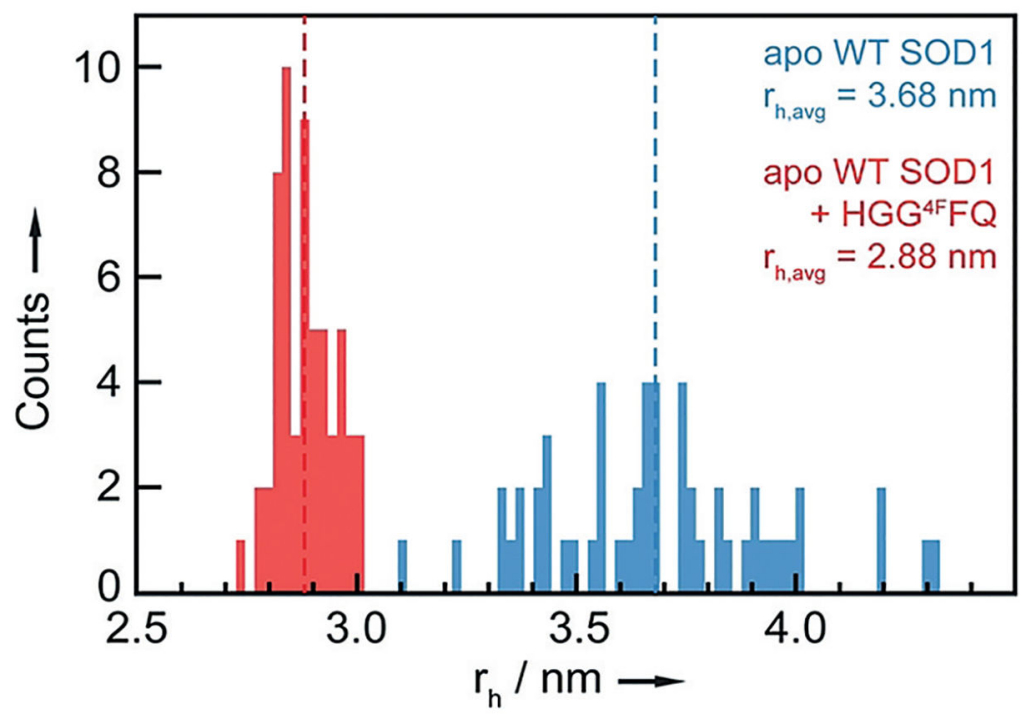

Figure 4.

Decreases in the hydrodynamic radius of apo WT SOD1 $\left(I_{\mathrm{h}}\right)$ are observed in the presence of $\mathrm{HGG}^{4 \mathrm{~F}} \mathrm{FQ}$. The distribution of these values also narrows with addition of ligand $(n=54)$. 


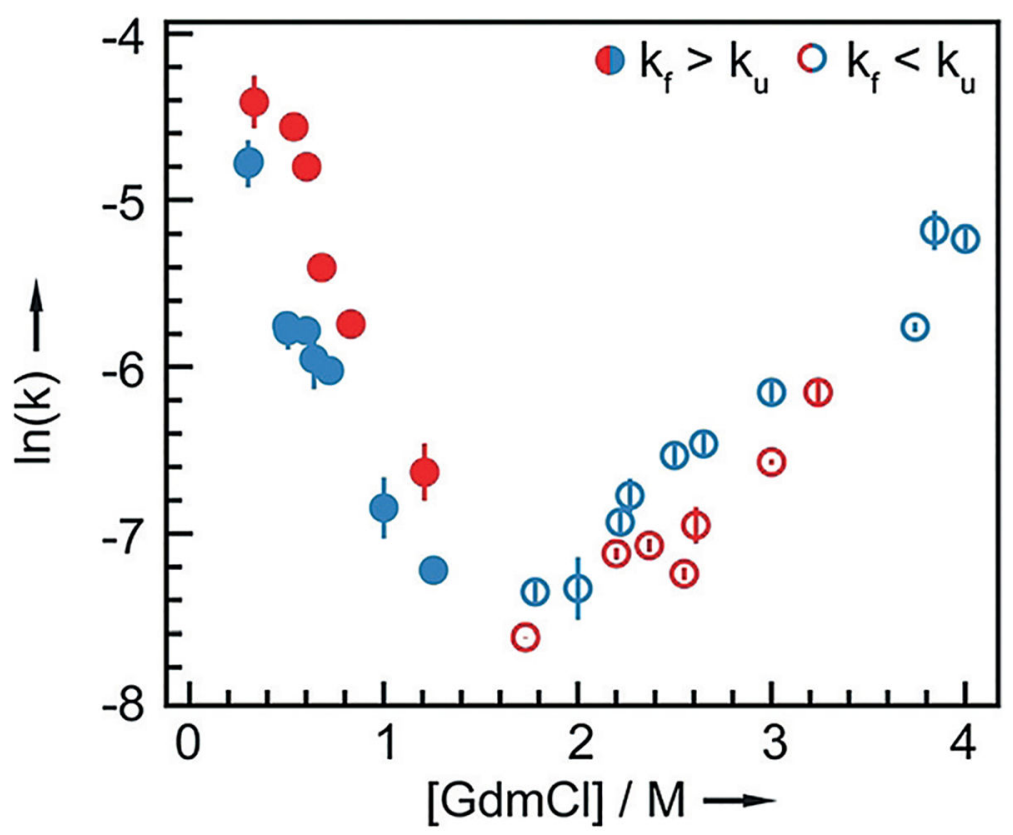

Figure 5.

Folding rate constants for apo WT SOD1, combined into a chevron plot, reveal an enhancement in the folding rate in the presence of ligand by a factor of 2.48 , without changing the rate-limiting transition structure $(p<0.005)$. 